Esta obra está bajo una Licencia Creative Commons Atribución-NoComercial-Compartirlgual 4.0 Internacional

(c) (i) (5) (2)

Experiencias innovadoras TIC en educación no formal

Agustín Zanotti y Mauricio Alejandro Grasso

DOI: https://doi.org/10.24215/16696581e261

\title{
Experiencias innovadoras TIC en educación no formal
}

\section{Innovative ICT experiences in non-formal education}

\author{
Agustín Zanotti \\ agustinzanotti@gmail.com \\ https://orcid.org/0000-0002-7662-7593 \\ Universidad Nacional de Villa María, Argentina \\ Mauricio Alejandro Grasso \\ mgrasso@unvm.edu.ar \\ https://orcid.org/0000-0002-0734-6616 \\ Universidad Nacional de Villa María, Argentina
}




\section{Resumen}

El artículo reúne avances de investigación sobre experiencias innovadoras con tecnologías en educación informal, en espacios autogestionados con apoyo de actores locales. Desde una perspectiva constructivista-crítica de la tecnología, buscamos establecer una vinculación entre modelos pedagógicos, mediaciones tecnológicas y prácticas de aprendizaje.

Analizamos dos experiencias educativas realizadas en la provincia de Córdoba. Los casos son la Tecnoteca municipal de la ciudad de Villa María y el club de programación CoderDojo radicado en la ciudad de Córdoba. Las mismas serán abordadas mediante una estrategia cualitativa que busca comprender y visibilizar dinámicas de innovación educativa.

Los resultados permiten valorar ciertas pedagogías de experimentación con tecnologías, aprendizajes basados en proyectos y equipos, así como participación en concursos, al tiempo que presentan el desafío de pensar articulaciones con el espacio escolar.

Palabras Clave: educación informal, educación digital, tecnologías digitales, apropiación de tecnologías

\section{Abstract}

The article gathers research advances on innovative experiences with technologies in informal education, in self-managed spaces with the support of local actors. From a constructivist-critical perspective of technology, we seek to establish a link between pedagogical models, technological mediations and learning practices.

We analyze two educational experiences carried out in the province of Córdoba. The cases are the Municipal Technology Lab of the city of Villa María and the CoderDojo programming club based in the city of Córdoba. They will be addressed through a qualitative strategy that seeks to understand and make visible dynamics of educational innovation.

The results allow to evaluate certain pedagogies of experimentation with technologies, learning based on projects and equipment, as well as participation in competitions, while presenting the challenge of thinking articulations with the school space.

Keywords: informal education, digital education, digital technologies, appropriation of technologies 
Introducción

Durante los últimos 30 años se han impulsado numerosas iniciativas públicas y privadas de educación digital, con el objetivo de superar su siempre diagnosticada crisis y adecuar el sistema educativo tradicional a una transformación constante de la mano de las Tecnologías de la Información y la Comunicación (TIC). Diferentes autores cuestionan el desacople de los rígidos muros escolares en tiempos de mediatización, volviendo la mirada hacia los cuerpos y las subjetividades (Sibilia, 2012), o hacia lo que interrumpe la homogeneidad del aula y los cambios en los modos de aprender y enseñar (Dussel, 2011; Feenberg, 2019a). Ello dentro de un imaginario inagotable de promesa digital, donde las tecnologías se asocian a un futuro ineludible de nuevas posibilidades (Cabrera, 2007).

En instancias previas de investigación, se abordaron modos de comunicación y educación mediados por tecnologías en la escuela secundaria ${ }^{1}$. El análisis partió de una perspectiva constructivista-crítica de la tecnología (Feenberg, 2012), buscando establecer una vinculación entre modelos pedagógicos, mediaciones tecnológicas y prácticas de aprendizaje. Prestamos especial atención al programa de inclusión digital socioeducativa Conectar Igualdad, el cual implicó un amplio despliegue de dispositivos e infraestructura con el objetivo de reducir la brecha digital. Nos centramos en procesos de apropiación de tecnologías (Grasso y Zanotti, 2014), en visiones y representaciones asociadas a las TIC (Zanotti y Arana, 2015) y consideraciones relativas a su implementación (Grasso, Pagola y Zanotti, 2016), tomando el aglomerado Villa María-Villa Nueva, en la provincia de Córdoba, Argentina.

Los avances de investigación nos permitieron observar que una parte de experiencias innovadoras con tecnologías estaban ocurriendo fuera de la escuela, en espacios autogestionados o con apoyos externos al sistema educativo formal. En ese sentido reconocemos que en reiteradas oportunidades, las instituciones escolares fueron permeables a experiencias de trabajo colaborativo y significativo con tecnologías, propias de comunidades de aprendizaje que cuestionan los límites de las aulas, la rigidez curricular y cierto mandato de imposibilidad técnica. En función de ello, decidimos enfocarnos en propuestas de aprendizaje informal, que mantienen sin embargo conexiones con trayectos formales.

1 Los proyectos son: Paradigmas tecnológicos libres, recursos educativos abiertos y apropiación de la tecnología. Prácticas de aprendizaje de jóvenes escolarizados en Córdoba; Tecnologías y prácticas de aprendizaje. Apropiación social y disputa entre los sujetos de la escuela secundaria; Alfabetizaciones, Tecnologías y Prácticas Educativas. Los nuevos modos de comunicar y sus significaciones en docentes de Nivel Medio. Universidad Nacional de Villa María. Res. 03/2016 I.I.; 479/14; 220/12. 
Siguiendo a Jenkins y otros:

\begin{abstract}
Mientras la educación formal es a menudo conservadora, el aprendizaje informal dentro de la cultura popular es a menudo experimental. Mientras la educación formal es estática, el aprendizaje informal es innovador. Las estructuras que sostienen el aprendizaje informal son más provisionales, las que apoyan la educación formal son más institucionales. Las comunidades de aprendizaje informal pueden evolucionar para responder a necesidades a corto plazo e intereses temporales, mientras las instituciones de educación pública no han cambiado mucho a pesar de décadas de reforma escolar. (2009, p. 9, Traducción propia)
\end{abstract}

A lo largo del artículo, nos detenemos en dos experiencias realizadas en la provincia de Córdoba. Los casos son la Tecnoteca municipal de la ciudad de Villa María y el Proyecto CoderDojo radicado en la ciudad de Córdoba. Las mismas serán abordadas desde un diseño flexible, mediante una estrategia cualitativa que busca comprender y visibilizar experiencias significativas.

\title{
Aproximación metodológica
}

Los casos y colectivos que conforman la muestra fueron seleccionados en el marco de un abordaje exploratorio basado en un mapeo y registro de experiencias innovadoras con TIC en educación no formal en la provincia. Para ello, se realizaron búsquedas de fuentes periodísticas, portales institucionales y plataformas de redes sociales, así como entrevistas a informantes clave y participantes de los proyectos seleccionados.

Buscamos con ello contribuir a una sociología de las ausencias, colocando la mirada en experiencias emergentes que, en el sentido de Boaventura de Sousa Santos (2003), permanecen invisibilizadas o incomprendidas para las ciencias sociales:

lo más preocupante en el mundo de hoy es que tanta experiencia social queda desperdiciada, debido a que ocurre en lugares remotos. Experiencias muy locales, no muy conocidas ni legitimadas por las ciencias sociales hegemónicas, son hostilizadas por los medios de comunicación social, y por eso han permanecido invisibles, "descredibilizadas". (...) Este concepto de razón metonímica contrae el presente porque deja por fuera mucha realidad, mucha experiencia, y al dejarlas afuera, al tornarlas invisibles, desperdicia la experiencia (p. 19-20).

El período analizado corresponde a los años 2017 y 2018. Vale mencionar que algunos integrantes de tales proyectos se incorporaron al equipo de investigación en el último periodo, de modo de ajustar el proceso a sus propios fines, fortalecer las iniciativas y realizar actividades conjuntas, en un esfuerzo de investigación-acción (Elliott, 1990). 
Innovación y experimentación en educación no formal

De la mano de Castells (1996) podemos definir las sociedades actuales como una era de la información, donde las TIC devienen una fuente principal de productividad y poder. Van Dijck (2016) se refiere en un sentido similar a una cultura de la conectividad, donde nuestras interacciones son crecientemente mediadas por poderosas plataformas de software social.

Entendiendo que la educación tiene la misión fundamental de habilitar una participación plena en la vida pública, comunitaria, ciudadana y económica, Jenkins et al. (2009) se preguntan por cuales son las capacidades requeridas para formar parte de lo que definen como una cultura participativa. En ella, los jóvenes asumen crecientemente un lugar de prosumidores en medios digitales, a través de diferentes registros como blogs, fotos, historias, videos, remixes de contenidos, y más.

Las nuevas habilidades propuestas pueden resumirse en las siguientes: experimentación y juego; improvisación y el descubrimiento; simulación; muestreo y mezcla de contenido; multitarea; cognición distribuida; inteligencia colectiva; ponderación de fuentes de información; navegación transmedia, sintetizar y diseminar información; discernir entre perspectivas y normas alternativas (Jenkins et al., 2009, p. 4)

En una lectura crítica, Fuchs (2017) argumenta que estas capacidades se orientan más al mundo del trabajo que a la vida cívica. Agrega la capacidad de pensamiento crítico, la cual supone un principio de comprensión sobre las formas de propiedad, las políticas y los modelos de negocio que subyacen a las herramientas y plataformas de software. En un sentido similar, Van Dijck (2016), asegura que los usuarios mantienen una capacidad de agencia y despliegan tácticas que influyen en los desarrollos tecnológicos.

Siguiendo a Feenberg (2019b), debemos entender a Internet y las plataformas de software social como parte integral del mundo de la vida contemporáneo. Este se presenta como un todo inacabado y en constante transformación. En la medida que tales procesos afectan de un modo directo nuestra vida cotidiana, es menester que la educación juegue un rol como espacio de reflexión colectiva y experimentación crítica. Dussel (2016), vuelve a poner en este punto el énfasis en el papel de la escuela en la sociedad contemporánea, como el lugar de lo difícil pero importante.

Al igual que otras políticas públicas que siguieron el modelo 1 a 1 en la región, el Programa Conectar Igualdad concentró una parte importante de sus esfuerzos en la entrega masiva de dispositivos, en este caso netbooks, las cuales se complementaban con conectividad e infraestructura escolar. Si bien el Programa contempló desde un inicio una serie de 
herramientas y estrategias de enseñanza y aprendizaje, estas fueron cambiando y ajustándose a lo largo del tiempo. Algunas definiciones, como el tipo de sistemas a utilizar, oscilaron en diferentes momentos (Grasso, Pagola y Zanotti, 2016).

Su reemplazo en 2017 por el Plan Aprender Conectados, se acompañó de un nuevo diagnóstico, centrado en la necesidad de promover habilidades digitales, en sintonía con la Agenda 2030 para el Desarrollo Sostenible de la ONU. Por otra parte, de una política universal se pasa a un diseño focalizado por proyectos, con énfasis en la innovación y el desarrollo de competencias (Ministerio de Educación, 2017a; 2017b).

Algunas dinámicas de trabajo colaborativo entre pares, diseño por proyectos, o espacios de experimentación y juego, existen desde hace tiempo en espacios no formales y son fuertemente estructuradores de tales experiencias. Podemos definir a la educación no formal como «toda actividad organizada, sistemática, educativa, realizada fuera del marco del sistema oficial, para facilitar determinadas clases de aprendizaje a subgrupos particulares de la población» (Coombs y Ahmed, 1974). Cobo y Moravec (2011) ubican a la educación no formal dentro de una nueva ecología de la educación, donde se relaciona a conceptos como aprendizajes invisibles, aprendizaje permanente educación expandida, edupunk, aprendizaje serendípico, ubicuo, entre otros.

Otra de las discusiones que rodean las políticas de inclusión digital educativa se refieren a las brechas de acceso, uso y apropiación. Nielsen (2006) distingue entre brecha económica, brecha de usabilidad y brecha de empoderamiento. Siguiendo el planteo del autor, mientras que las brechas referidas al acceso y uso están siendo crecientemente afrontadas, la restante requerirá de una estrategia integral hacia el futuro:

En definitiva, soy muy optimista acerca de la brecha económica, que está desapareciendo rápidamente en los países industrializados. La brecha de usabilidad necesitará más tiempo para cerrarse pero al menos sabemos cómo manejarla, es simplemente una cuestión de decidir hacerlo. Soy muy pesimista, sin embargo, sobre la brecha de empoderamiento, la cual considero que se tornará más severa en el futuro. (Nielsen, 2006, Traducción propia)

Sobre la base de tales definiciones, incorporamos el concepto de apropiación de Aguerre et. al., quienes se refieren a:

el proceso material y simbólico de interpretación y dotación de sentido respecto a un determinado artefacto cultural por parte de un grupo social. (...) El concepto de apropiación pone el énfasis entonces en la capacidad de los sujetos para volverlas significativas de acuerdo a sus propios propósitos. (Aguerre et. al., 2010, p. 11) 
Si bien se comprende que relación con la brecha económica, en nuestro país y región no está saldada, nos interesa detenernos en la relación que establece el autor sobre los demás aspectos. Con todo ello podemos decir que la apropiación es, un proceso hermenéutico relacional de interpretación y dotación de sentido que se genera en un contexto socio-histórico particular, en el cual intervienen la pertenencia socio-cultural de los sujetos y los universos simbólicos previos, propios de cada grupo, desde donde las tecnologías son incorporadas (Zanotti y Arana, 2015).

Dicho conjunto de herramientas y recursos representan o reflejan prácticas asociadas con un paradigma tecnológico libre (Pagola, 2010a). Entendemos al mismo como el conjunto de las producciones digitales (hardware, software y obras culturales) así como sus prácticas de creación y circulación; y las conceptualizaciones asociadas, en las cuales se definen las libertades de los usuarios como derechos que deben ser garantizados (el derecho a la cultura, a la información, a la libertad de expresión, a la privacidad, principalmente) por encima de cualquier otro tipo de factores (económicos, de seguridad, u otros). Este se encuentra posibilitado por el desarrollo de las TIC y numerosas plataformas de colaboración entre pares (Kostakis y Bauwens, 2014). Desarrollos que constituyen y definen este paradigma son: el software libre, las obras culturales libres y los recursos educativos abiertos, entre los más relevantes.

En función de las investigaciones previas, consideramos que los desarrollos del paradigma tecnológico libre supone dos aspectos centrales:

- la superación de la visión instrumental de la tecnología (y el concepto de neutralidad tecnológica, entre otros), para dar lugar a usos informados y críticos.

- la reflexión y cuestionamiento sobre la dimensión política inscripta en el fenómeno técnico y los imaginarios sociales que se desarrollan en torno a su introducción y uso masivo.

Tales manifestaciones se enmarcan en la disputa por los bienes informacionales como bienes comunes. El nuevo movimiento de los comunes «se carga con energía haciendo señas a los ciudadanos del mundo para desarrollar nuevas formas de autogobierno, colaboración y acción colectiva» (Hess, 2008, p. 4). Estas manifestaciones son particularmente visibles en la web y señala una problematización en relación con lo que es o debería ser compartido en el mundo que nos rodea, la importancia de comprender quién comparte qué, cómo lo compartimos y cómo sostenemos los comunes para las generaciones futuras. 
Análisis de casos

\section{Caso 1: Tecnoteca Municipal Villa María}

El proyecto Tecnoteca Municipal Villa María se inicia en 2011, con un equipo multidisciplinario. Los impulsores buscaron conformar un espacio educativo y cultural en el centro de la ciudad, enclavado en un predio de edificios recuperados de viejos talleres del ferrocarril. El proyecto, toma como fuente de inspiración otras iniciativas pioneras en el país, como Puerta $18^{2}$ y el Museo Participativo de Ciencias ${ }^{3}$. En la actualidad, en la tecnoteca, trabajan unas 20 personas entre profesores de computación, mecatrónica, diseño y producción de imagen, educación física y psicopedagogía (La voz de San Justo, 2017). Con ello se ofrece una nutrida grilla de actividades para personas de todas las edades.

Públicamente, la Tecnoteca se define como un espacio abierto basado en la innovación tecnológica y los procesos productivos. Se trabaja de acuerdo con 4 movimientos, que sirven como guías o hilos conductores: cultura digital, cultura libre, cultura maker, cultura hacker (Educ.ar, 2016). Todas las actividades buscan articular con instituciones educativas y sociales de la ciudad y la región. Se busca con ello que los contenidos se integren a problemáticas abordadas desde la escuela o en diferentes campañas. Entre las actividades que ofrece la se encuentran: experiencia robótica, Lego, edición de video, micro cine 3D, producción de contenidos periodísticos, realidad virtual, sala infantil, sala de radio, software para procesos productivos (Educ.ar, 2015).

Con el paso del tiempo, sus proyectos fueron evolucionando en diferentes direcciones. Aunque el estudio de televisión no cuenta con canal propio, provee de programación infantil a tres canales locales. Entrega un enlatado de 8 horas de programación gratuita cada semestre. Algunos contenidos se difunden asimismo por streaming, haciendo uso de nuevos formatos. El estudio de grabación permite que músicos locales puedan crear ediciones de manera gratuita. Además, se creó la radio FM 88.1, que cuenta con más de 35 programas locales educativos y culturales (La voz de San Justo, 20176 de febrero).

\footnotetext{
${ }^{2}$ Puerta 18 es un espacio gratuito para jóvenes de 13 a 24 años, fundado en 2007, donde se estimulan habilidades y vocaciones a través del uso de diversas herramientas tecnológicas. Los jóvenes desarrollan proyectos de video, diseño, robótica, animación, música entre otros. Es miembro de The Clubhouse Network. (Puerta 18, 2017).

${ }^{3}$ El Museo Participativo de Ciencias (MPC) es una institución dedicada a la divulgación de las ciencias en forma lúdica y participativa. Es una propuesta activa para público de todas las edades. abrió sus puertas en 1988 y a la fecha ha sido visitado por más de dos millones de personas. Está basado en la filosofía del "prohibido no tocar" y "aprender haciendo" (MPC, 2017). 
Los trabajos con robótica adquirieron especial repercusión a nivel regional. Sobre la base de un diseño basado en proyectos, se dictaron talleres específicos de robótica en relación con temas ecológicos (accidentes de contaminación ambiental, recuperación de residuos sólidos urbanos con latitas de gaseosa, entre otros). Se busca que las propuestas articulen con contenidos de manera transversal entre diferentes áreas de conocimiento. Ejemplo de ello fue el proyecto de construcción de un alacrán robótico, al que se le sumó un taller sobre la prevención de picaduras de alacranes. Asimismo, realizaron impresiones 3D de manos humanas, utilizando un filamento que se hace con plástico PET (reciclado de botellas de gaseosas).

La Tecnoteca realizó además las primeras prótesis 3D en la provincia. Hasta el momento se imprimieron 10 manos ortopédicas y se investiga sobre nuevos modelos y materiales flexibles que evitan la utilización de piezas metálicas en articulaciones, haciéndolas más livianas y estéticas. Actualmente, se continúa en la fabricación de manos y brazos a partir de impresión 3D, pero con motores eléctricos y sensores musculares. Se busca asimismo que la persona destinataria se involucre activamente del proceso, tomando decisiones y aprendiendo a producir su propia prótesis (El diario del centro del país, 201619 de marzo).

El área de informática ofrece cursos y talleres de capacitación en usos desde básicos hasta avanzados. Se utilizan herramientas libres y educativas. Se ha participado en certámenes de programación y robótica. Desde 2016 de la Global Game Jam, una competencia descentralizada global de creación de videojuegos (GGJ, 2019). La Tecnoteca fue asimismo el representante provincial en la Copa Robótica 2019:

\begin{abstract}
el robot estuvo muy bien logrado. Se nos presentaron algunas dificultades técnicas y hubieran sido fácilmente solucionadas si el equipo hubiese logrado una mayor maduración. Nos faltó tiempo de cohesión. Trabajé mucho en mi rol en formar un equipo, pero el tiempo fue acotado. Así que frente a dificultades halladas en el camino no pudimos trabajarlas de forma plena desde una concepción de equipo maduro. Esto afectó en la competencia dado que no pudimos resolver un problema que surgió en CABA con el mecanismo de tiro. Eso sí, volvieron mucho más maduros en equipo. (Cierra, 2019)
\end{abstract}

Con ello queremos rescatar la experiencia del trabajo colaborativo, como una de las principales características de los casos analizados.

\title{
Un perfil en construcción
}

Observamos que el proyecto Tecnoteca fue construyendo sobre la marcha un perfil como espacio de innovación tecnológica y cultural, buscando su lugar específico en un entorno en el 
que existían y se irían incorporando otras iniciativas vinculadas a la capacitación en nuevas tecnologías.

Según entrevistados, esta necesidad de definición se vio desde el momento mismo de su creación. Luego de un periodo inicial de fuerte impacto en 2011, en la que muchas personas se acercaron a conocer la novedosa propuesta, la concurrencia en 2012 y 2013 comenzó a aminorar. Fue por ello necesario buscar formas de articulación, en especial con las escuelas primarias y secundarias de la localidad.

\begin{abstract}
Lo que tuvimos que hacer fue salir a las escuelas a buscar a los alumnos para hacer cursos fuera del horario de clase. Los maestros no querían venir, no querían exponerse ellos ni exponer a los chicos. (...). Hicimos los primeros talleres de robótica, fueron muy exitosos, volvieron a las aulas y comentaron lo fantástico que era. Entonces cuando volvimos por segunda vez las maestras ya me estaban esperando, ya querían ir con todo el grupo. Al siguiente año repetimos y tuvimos como 1600 alumnos de primero a sexto grado de escuela primaria. (Entrevista Tecnoteca, 2017)
\end{abstract}

Más allá de los talleres, la tecnoteca ofrece la posibilidad de una modalidad flexible a demanda, donde las actividades se adaptan a la currícula de las asignaturas interesadas, de manera trabajar en un propuesta conjunta.

\begin{abstract}
El objetivo era que un docente viniera y diera cualquier actividad que estuviera haciendo, y nosotros le pusiéramos algo más de tecnología. Para que a los chicos les resultara también más atractivo. Nos habíamos comprometido a que investigábamos el tema y en 48 horas les presentábamos la actividad. Pero realmente eran pocos los que venían. Entonces fuimos a buscarlos ya con una propuesta. Eran muchas las propuestas que podíamos hacer, pero diseñamos cuatro o cinco concretas. (Entrevista Tecnoteca, 2017)
\end{abstract}

La tecnoteca busca de este modo articular con las necesidades de las instituciones educativas. También fue sede de un encuentro de Conectar Igualdad en 2013. Este recibió a las escuelas de la región junto a talleristas y equipos del programa nacional. El encuentro sirvió para ampliar los contactos con la comunidad educativa y un sector vinculado al mismo tiempo con la comunidad de software libre local, que estaría luego comprometida en la puesta en marcha del sistema operativo libre Huayra.

\title{
Usuarios y productores
}

Algunos entrevistados insisten en una función de la Tecnoteca fuertemente ligada a la apropiación de la tecnología, generando capacidades y apoyo institucional que puedan luego servir para la satisfacción de necesidades, el emprendedorismo y un empoderamiento respecto 
a las tecnologías. Esta visión busca superar el lugar del mero usuario de tecnologías, o una finalidad de estas vinculadas al ocio y el entretenimiento.

Mi formación que tenía que ver con emprendedorismo, con software libre, con Linux, con todas esas cosas. En realidad eran todas herramientas para que pudieran ser independientes y hacer lo que sea, lo que quisieras hacer. De ahí venía mi formación y eso es lo que apliqué en mi área, que era informática y robótica. A la gente le parecieron re buenas todas las ideas, (...) básicamente te empoderaban de cosas para que pudieras lograr lo que vos quisieras. (Entrevista Tecnoteca, 2017)

Se promueve una dinámica tallerista donde la filosofía del aprender haciendo y la dilución de las fronteras entre el juego y la actividad productiva tienen lugar (Lund, 2017). Las decisiones respecto a qué tecnologías usar, o qué tecnologías habilitan de la mejor manera estas posibilidades, se plantean así en varias oportunidades, revelando la no-neutralidad de ciertos diseños tecno-educativos, así como las posibilidades de una lectura constructivista-crítica:

\begin{abstract}
Armamos un robot con alumnos de quinto grado. Este detectaba el sonido de la voz del docente cuando gritaba mucho y levantaba una banderita para que se callara. Presentamos el proyecto en la feria de ciencias y les fue muy mal. Estudiaron, investigaron todo, desde mi punto de vista era un proyecto ejemplar. Cuando preguntamos cuál fue el veredicto, nos dijeron que la mala nota que les pusieron tenía que ver con que usaron robot LEGO, y que ya venía todo hecho. (...) Entonces al año siguiente quisimos hacer lo mismo pero con hardware libre. Nos contactamos con gente de la comunidad que nos ayudó, los chicos soldaron la placa e hicieron todo. Lo construimos con basura digital. El robot hacía lo mismo que el año anterior, detectaba el sonido y todo. Este proyecto lo presentamos en Buenos Aires, a Conectar Igualdad, y les encantó. (Entrevista Tecnoteca, 2017)
\end{abstract}

La experiencia acumulada en torno a las tecnologías libres, hicieron que desde 2014 la Tecnoteca se propusiera como sede local organizadora del Festival Latinoamericano de Software Libre (FLISOL), un encuentro que se realiza anualmente y busca poner en común y sumar nuevos participantes a su comunidad regional. Algunos miembros de la Tecnoteca realizaron asimismo migraciones a software libre en escuelas.

Es necesario remarcar que las decisiones que van moldeando el perfil de la Tecnoteca no han estado exentas de conflictos. En este sentido, los entrevistados señalan diferencias en las visiones y los abordajes entre las diferentes áreas, discontinuidad de proyectos e inclusive condicionamientos políticos o gubernamentales. Ellos no han impedido un crecimiento y despliegue del proyecto hasta la actualidad, tanto en la cantidad de personas, en su mayoría jóvenes, que participan de las actividades, como en el alcance de su convocatoria. 


\section{Caso 2: Proyecto CoderDojo Córdoba}

CoderDojo es un proyecto internacional sin ánimo de lucro para la formación de clubes de programación donde niños y jóvenes entre 7 y 17 años tengan la posibilidad de aprender a través de un sistema de educación abierto e informal. Su principal objetivo consiste en transmitir los conocimientos necesarios sobre programación partiendo de los intereses propios de cada niño y respetando sus ritmos de aprendizaje.

CoderDojo nace en Irlanda en 2011 y se encuentra funcionando en diversos países, con 12,000 voluntarios a nivel global. Para ello se creó en 2013 la CoderDojo Foundation (CoderDojo, 2019). En Argentina, el primero de sus clubes se conformó en la ciudad de Córdoba. Es un proyecto autogestionado donde programadores voluntarios, con el apoyo de padres, guían el aprendizaje y se ocupan de la organización. En la actualidad funciona en espacios cedidos por la Municipalidad en el Parque Educativo Sur y en la Universidad Tecnológica Nacional (UTN). En 2019 alcanzó los 150 encuentros, que se realizan los días sábados (Coder Dojo Córdoba, 2019).

A cada centro acuden niños y jóvenes de distintos barrios, en su mayoría de bajos recursos. «Es muy difícil para alguien que vive en un asentamiento o en una situación muy precaria acceder a este tipo de entorno, de amigos, de conocidos, de conocimientos, de facilidad para algo» (Entrevista CoderDojo Córdoba, 2017). Se busca que tales aprendizajes contribuyan a ampliar los horizontes de expectativas en su formación, para una posterior inserción laboral:

\footnotetext{
El participar en esto, aunque sea como un juego, te abre la cabeza y te abre las posibilidades, aunque sean mentales, de que vos podes hacer otra cosa, de que vos podes superar las limitaciones en las que vivís. Si nadie te ofrece una salida, las salidas no son mágicas, no podés inventarte cosas en el aire. (Entrevista CoderDojo Córdoba, 2017)
}

En cuanto a la organización de las clases existen diferencias con el sistema educativo tradicional. Se organizan grupos de trabajo sobre ejes temáticos particulares y cada niño que se incorpora se une a ellos de acuerdo a su interés. La edad no es en este caso un factor de clasificación sino los intereses en función de proyectos. Esto permite que «los chicos desmitifiquen la creencia de que por ser más grande se sabe más o de que si alguien es más chico no puede saber más sobre algo».

El proyecto presta especial atención a la brecha de género que se perpetúa en la formación tecnológica y de software, Esta cuestión está asociada a una serie de imaginarios culturales existentes acerca de lo tecnológico como una actividad propia de varones, que repercute en el 
conjunto de la industria tecnológica (Pagola, 2010b). Si bien por parte del equipo se incentiva su participación de mujeres, niñas y adolescentes, no se ha logrado que supere el $25 \%$.

\section{Aprendizaje por proyectos}

CoderDojo se propone enseñar a programar desde una lógica no tradicional. En lugar de transmitir cuestiones teóricas, la idea es que los niños aprendan a programar a través de sus propias necesidades, aplicando el conocimiento a cuestiones prácticas. Por este motivo no es un requisito poseer conocimientos previos, lo cual posibilita aún más la inclusión de una diversidad de jóvenes.

El hardware y software utilizado es abierto, lo cual favorece su reimplementación y reutilización. Entre las opciones de desarrollo encontramos a Scratch, un lenguaje de programación visual desarrollado por MIT Media Lab. Su principal característica consiste en que permite el aprendizaje de la programación y el pensamiento computacional sin tener conocimientos profundos sobre el código. También se utiliza HTML/CSS, Python, Unity, Sonic PI, Blocks CAD, FreeCAD, Wolfram, Blender, App Inventor, entre otras posibilidades.

Para ello mantiene una repositorio de proyectos educativos que pueden replicarse en cada club. Los temas disponibles son variados, incluyendo arte, ciberseguridad, espacio, fotografía, humor, juegos, libros, meteorología, música, robótica, tecnología usable y más. Para ello pueden incorporarse componentes de hardware, electrónica, sensores, placas computadoras Raspberry Pi o impresoras 3D (CoderDojo, 2019).

Ello genera un gran estímulo en ellos ya que no se aburren, algo que a esa edad suele suceder. En CoderDojo no está prohibido hacer nada y por es los jóvenes se sienten libres. La idea es que se planteen un objetivo, algo que quieren resolver y que puedan probar distintas técnicas y comprobar así cuales resultan útiles para cada caso. Uno de los voluntarios entrevistados dice que:

Los chicos aprenden también a elegir, a preguntarse qué les sirve, que les es más útil para ese propósito, a no quedarse con la idea de que aprenden algo y lo pueden usar para todo, se vuelven mucho más prácticos y también cambian de herramientas como de pantalones, por decir una cosa, porque lo que ellos buscan es hacer algo, no importa con qué lo hacen. (Entrevista CoderDojo Córdoba, 2017)

Esta modalidad particular de enseñanza genera también una superación del miedo a la frustración y la equivocación que los niños desarrollan comúnmente. Dicho miedo funciona en ocasiones como un límite a su creatividad que es lo que los lleva a inventar y probar distintas 
opciones. De este modo, los niños aprenden a programar jugando y tratando de solucionar los distintos problemas que se les presentan en el desarrollo de dicho juego.

(...) una de las cosas que más los bloquea a los chicos es el fracasar, el equivocarse y en realidad cuando uno programa se equivoca un montón de veces, en realidad cuando hace cualquier cosa. Pero la historia es aprender a superarlo y ver qué hiciste mal o qué hizo mal el grupo, no quedarse sobre eso y buscar una salida, una solución, y es lo que tratamos de hacer. (Entrevista CoderDojo Córdoba, 2017)

Trabajando bajo esta secuencia o estrategia didáctica, a partir de una necesidad que es identificada y complejizada por los chicos es que se ha logrado desarrollar prototipos orientadas a solución de problemas del entorno. Algunos ejemplos diseñados localmente son un chaleco con sensores que funciones como asistente para personas con discapacidad visual. El mismo está pensado de modo que identifique los obstáculos que pueden existir para estas personas en la vía pública y transmitírselo a través de auriculares. Este proyecto recibió donaciones de dinero y de materiales, para los ensayos con los primeros prototipos (Entrevista CoderDojo Córdoba, 2017). De este modo, con el paso del tiempo y el conocimiento de las actividades que el grupo realiza se logra constituir una experiencia más acabada y productiva. Otro hito en el proyecto fue la participación en certámenes de programación a nivel internacional. Para ello se trabajaron diferentes proyectos a lo largo de los encuentros y se organizaron campañas de difusión, búsqueda de sponsors y crowdsourcing. Hasta el momento niños y jóvenes participantes asistieron a ediciones del evento global en Irlanda Coolest Projects y al hackatón Space Apps Challenge en su edición local.

\section{Consideraciones finales}

La comparación entre los casos nos permite establecer ciertas similitudes en los procesos y estrategias de los actores que los llevaron a cabo. A la vez, esas experiencias y prácticas interpelan al sistema educativo formal, en virtud de las dimensiones o límites del aula, canales de circulación, promoción y producción de saberes y desde las posibilidades de los aprendizajes. En ese sentido es menester preguntarse ¿Qué significado tiene en esto la apropiación social de las tecnologías? y, a la vez, ¿Esas mismas secuencias pueden aplicarse en otras circunstancias y contextos de aprendizaje?

El análisis y breve exposición de los casos intenta visibilizar posibilidades emergentes de educación digital. Pudimos apreciar que la relación entre escuela y contexto social, las experiencias de aprendizajes con y sobre tecnologías son dinámicas. Pusimos, en esta oportunidad, el foco en la educación informal y los procesos sociales que le dan sentido, lo 
cual implica poder apreciar aspectos relevantes y característicos de la cultura e instituciones contemporáneas.

Si nos detenemos en los elementos en común de los casos, podemos encontrar que proponen dinámicas innovadoras de aprender haciendo. Para ello, promueven la experimentación activa con tecnologías basadas en soluciones abiertas y de bajo costo, manteniendo entre sus metas la inclusión socioeducativa. Se basan además en aprendizajes por proyectos, incluyendo como elemento motivador la participación en certámenes nacionales e internacionales. En cuanto a su organización, combinan trabajo voluntario y apoyo del Estado (en ambos casos de nivel municipal).

Nos interesaron aquellos componentes que problematizan los usos de las tecnologías, así como aquellos que generan un sentido de empoderamiento y apropiación social. Con ello queremos rescatar la experiencia del trabajo colaborativo, como una de las características sobresalientes de los proyectos analizados. En relación a este punto, incorporamos a modo de cierre la reflexión de García Canclini respecto al rol de la educación para las futuras generaciones:

\begin{abstract}
Me parece excitante para las escuelas y universidades imaginar cómo conocer y actuar en estos nuevos escenarios. En ciertos casos, las redes sociales, especialmente entre los jóvenes, están logrando el paso de consumidores a prosumidores, de simples usuarios a creadores. Si se abren nuevas posibilidades de emancipación, las escuelas son claves para que nos emancipemos. Frente a la desciudadanización sistémica del capitalismo conectivo, la educación es necesaria para que las capacidades de los internautas no se limiten a mostrar creatividad y ocurrencias, sino para construir comunidades alternativas. (2019, 19 de septiembre).
\end{abstract}

\title{
Referencias bibliográficas
}

Aguerre, C., Larghi, S. B., Calamari, M., Fontecoba, A., Gaztañaga, M., Moguillansky, M., \& de León, J. P. (2010). La apropiación de las TIC por jóvenes de sectores populares urbanos en espacios de acceso público. Revista Argentina de Estudios de Juventud, $1(3)$.

Cabrera, D. (2007). La educación en el camino de las nuevas tecnologías. Razón y Palabra, 59. Recuperado de www.razonypalabra.org.mx

Castells, M. (1996). La era de la información: economía, sociedad y cultura (Vol. 1). Buenos aires: Siglo XXI. 
Cierra, G. (2019, 22 de agosto). Copa Robótica 2019. Saberes digitales aplicados a problemas de este tiempo. Reportaje a Gustavo Cierra. Recuperado de http://saberesdigitales.unipe.edu.ar/articulos/20-tecnoteca-municipalidad-de-villa-mariacordoba

Cobo, C. y Moravec, J. W. (2011). Aprendizaje invisible. Hacia una nueva ecología de la educación. Barcelona: Publicacions i Edicions Universitat de Barcelona.

Coombs, P. y Ahmed M. (1973). Perspectivas de UNESCO, III (3), 315-338.

De Sousa, B. (2003). Crítica de la razón indolente. Contra el desperdicio de la experiencia. Para un nuevo sentido común: La ciencia, el derecho y la política en la transición paradigmática, 1. Bilbao: Desclée de Brouwer.

Dussel, I. (2011). Aprender y enseñar en la cultura digital. Buenos Aires: Fundación Santillana. Recuperado de http://www.oei.org.ar/7BASICOp.pdf

Dussel, I. (2016). Inés Dussel. "La escuela debería ser el espacio de lo difícil pero importante". Recuperado de https://bit.ly/2YkCRfA

Elliott, J. (1990). La investigación-acción en educación. Madrid: Morata.

Feenberg, A. (2012). Transformar la tecnología. Una nueva visita a la teoría crítica. Bernal: Universidad Nacional de Quilmes.

Feenberg, A. (2019a). Postdigital or Predigital?. Postdigital Science and Education, 1(1), 8-9.

Feenberg, A. (2019b). The Internet as network, world, co-construction, and mode of governance. The Information Society, 1-15.

Fuchs, C. (2017). Social media: A critical introduction. London: Sage.

García Canclini, N. (2019, 19 de septiembre). Entrevista a Néstor García Canclini: La cultura digital cambia la lectura y los modos de estudiarla. Recuperado de https://bit.ly/38fdNef

Grasso, M., Pagola, L. y Zanotti, A. (2016). Políticas de inclusión digital en argentina. Usos y apropiaciones dentro y fuera de la escuela. Píxel-Bit. Revista de Medios y Educación, 49. doi: 10.12795/pixelbit.2017.i50.06

Grasso, M. y Zanotti, A. (2014). Discusiones sobre la relación entre las tecnologías y la sociedad. Aproximaciones desde el constructivismo tecnológico. En M. Aimino, G. Domjan y M. Grasso (Eds.). Los nuevos modos de comunicar: educación, tecnologías y escuela secundaria. Villa María: EdUViM.

Hess, C. (2008). Mapping the New Commons. Ponencia presentada en 12th Biennial Conference of the International Association for the Study of the Commons. England: University of Gloucestershire. 
Jenkins, H., Purushotma, R., Weigel, M., Clinton, K. y Robison, A. J. (2009). Confronting the challenges of participatory culture: Media education for the 21st century. Cambridge: MIT Press.

Kostakis, V. y Bauwens, M. (2014). Network society and future scenarios for a collaborative economy. Springer.

Ministerio de Educación de la Nación (2017a). Orientaciones pedagógicas de Educación Digital. Ciudad Autónoma de Buenos Aires: Ministerio de Educación de la Nación.

Ministerio de Educación de la Nación (2017b). Competencias de Educación Digital. Ciudad Autónoma de Buenos Aires: Ministerio de Educación de la Nación.

Pagola, L. (2010a). Efecto copyleft avant la lettre, o cómo explicar el copyleft donde todos lo practicamos. En B. Busaniche, et. al., Argentina Copyleft: la crisis del modelo de derecho de autor y las prácticas para democratizar la cultura. Córdoba: Fundación Vía Libre.

Pagola, L. (2010b). Sensibilización Tecnológica: mujeres construyendo la sociedad del conocimiento. En S. Santoro y S. Chaher, Las palabras tienen sexo II: herramientas para un periodismo de género. Buenos Aires: Artemisa Comunicación.

Sibilia, P. (2012). La escuela en un mundo hiperconectado: ¿redes en vez de muros?. Revista Educación y Pedagogía, 24 (62), enero-abril, 135-144.

Van Dijck, J. (2016). La cultura de la conectividad: una historia crítica de las redes sociales. Buenos aires: Siglo XXI.

Zanotti, A. y Arana, A. (2015). Implementación del Programa Conectar Igualdad en el aglomerado Villa María-Villa Nueva, Córdoba, Argentina. Ciencia, docencia y tecnología, (50), 120-143.

Sitios Web

Coder Dojo. (2019). Recuperado de https://www.coderdojo.com/

Coder Dojo Córdoba. (2019). Recuperado de https://www.coderdojocordoba.org/

Educ.ar (2015, 2 de septiembre). La Tecnoteca de Villa María. Recuperado de https://www.educ.ar/noticias/127847/la-tecnoteca-de-villa-maria

Educ.ar (2016, 8 de abril). La Tecnoteca de Villa María no para. Recuperado de https://www.educ.ar/noticias/130627/la-tecnoteca-de-villa-maria-no-para

FLISOL2015/Argentina/VillaMaria. (2019). Recuperado

de https://flisol.info/FLISOL2015/Argentina/VillaMaria 
GGJ Villa María. (2019). Recuperado de https://globalgamejam.org/2017/jam-sites/ggi-villamar\%C3\%ADa

La voz de San Justo (2017, 6 de febrero). La Tecnoteca de Villa María, un espacio modelo y de encuentro para los más chicos. Recuperado de https://bit.ly/2sU4Ebb

El diario del centro del país (2016, 19 de marzo). Una niña de 7 años recibió en la Tecnoteca una manito ortopédica. Recuperado de https://bit.ly/2LuokZm

MPC- Prohibido no tocar. Recuperado de 2017 de http://www.mpc.org.ar/home.htm 